\title{
Differences in psychiatric inpatients according to migrant status: a 2-year retrospective study
}

C. Miranda ${ }^{1}$, A.B. Medeiros ${ }^{1}$, A. Carapucinha ${ }^{1}$, A. Barcelos ${ }^{1}$, C. Fernandes Santos ${ }^{1}$, F. Fernandes Martins ${ }^{1}$, M. Bernardo ${ }^{1}$, T. Mendonça ${ }^{1}$, N. Descalço ${ }^{1}$, P. Casimiro ${ }^{1}$, V. Henriques ${ }^{1}$ E. Conde ${ }^{1}$, F. Gonçalves ${ }^{1}$

${ }^{1}$ Hospital Garcia de Orta, Department of Psychiatry, Almada, Portugal.

\section{OBJECTIVES}

- A brief report concerning differences in psychiatric inpatients according to place of birth

\section{BACKGROUND AND AIMS}

- The ethnic and migrant status is known to have significant impact on mental health

- Immigrants are subject to a number of stressors that can implicate on their mental health, including separation from their country of origin, family members, and culture, as well as having to navigate a new and unfamiliar culture and physical environment.

The present study aimed to evaluate the sociodemographic and clinical characteristics of Portuguese born versus inpatients born abroad.

\section{MATERIALS AND METHODS}

This study was conducted in the acute inpatient unit of a psychiatric service, integrated in a public general hospital. Hospital Garcia de Orta is located in the municipality of Almada and it serves a highly urban and multiethnic population of over 350.000 inhabitants of the municipalities of Almada and Seixal. Over $4,55 \%$ of residents are immigrants, about $40 \%$ from African Portuguesespeaking countries, such as Angola, Cape Verde and Guinea-Bissau, and 25,7\% from Brazil (according to the 2017's Census collected by the Portuguese institute of Statistics).

- Retrospective data for all patients admitted to the acute psychiatric unit from January 2016 to December 2017 were reviewed by using a computerized database.

- Sociodemographic and clinical characteristics of all inpatients admitted were collected included age, gender, education, labor and marital status, length of stay, history of substance abuse, compulsory admission and main diagnosis at discharge.

In light of the Portuguese constitutional law, it is not possible to collect ethnic or racial data. Therefore, the authors used the place of birth (Portugal versus Other Origins) as a proxy for migrant status - patients born outside of Portugal were compared with patients born in Portugal.

\section{RESULTS}

During this two-year period, there was a total of 776 psychiatric inpatient admissions, out of which 147 patients (19\%) were born outside of Portugal.

\section{GRAPHIC 1: PLACE OF BIRTH}
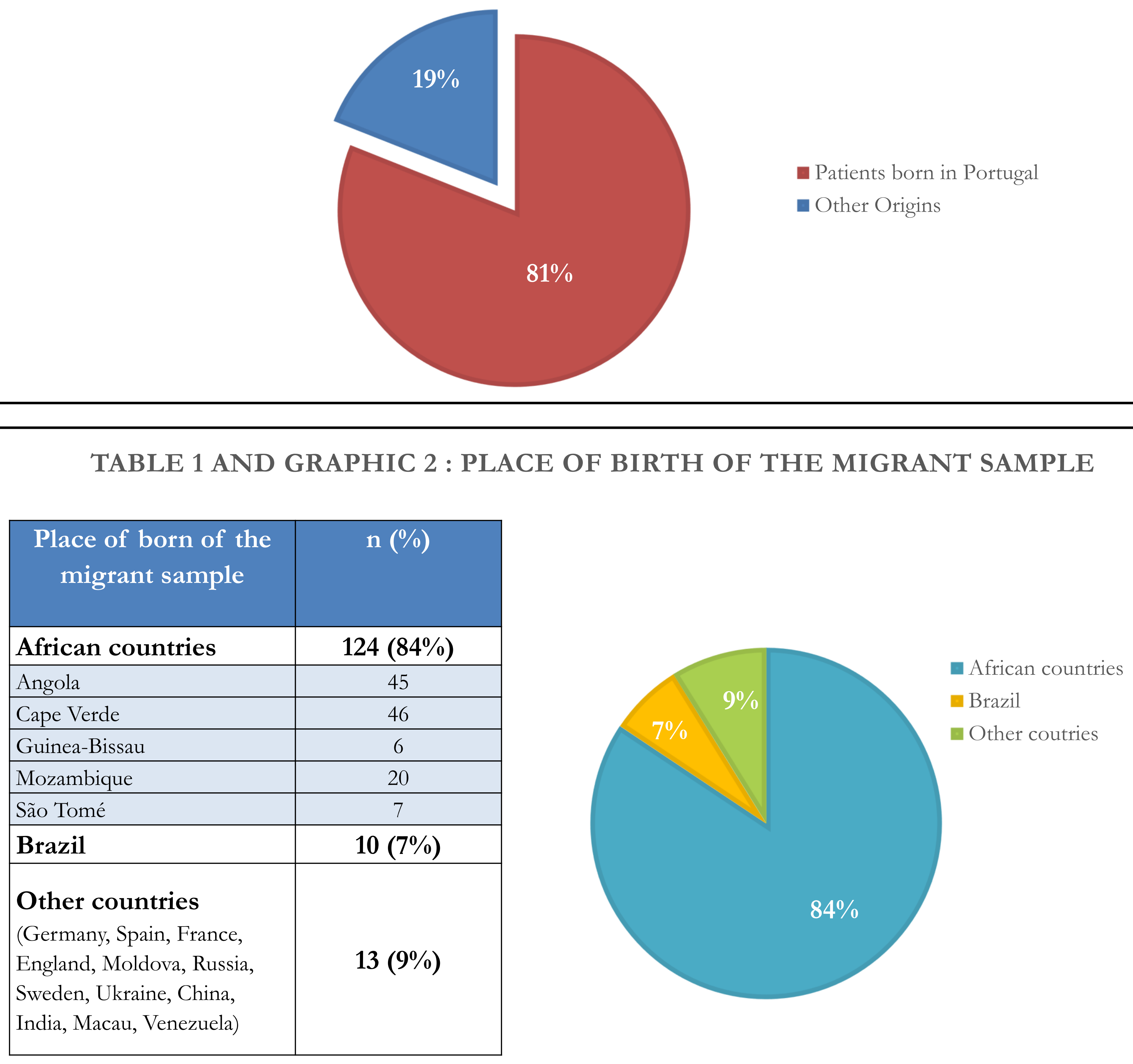

Patients born in African countries (Angola, Cape Verde, Guinea-Bissau, Mozambique and São Tomé and Príncipe) comprised $84 \%$ of those. Owing to the fact that this population represent the vast majority of the migrant sample, the autors decided to study and analyze this sub-population separately and to compare it with the Portuguese born inpatients.

\begin{tabular}{|c|c|c|}
\hline \multicolumn{3}{|c|}{ TABLE 2: SOCIODEMOGRAPHIC CHARACTERISTICS } \\
\hline & $\begin{array}{l}\text { Patients born in } \\
\text { Portugal }\end{array}$ & $\begin{array}{c}\text { Patients born outside of } \\
\text { Portugal }\end{array}$ \\
\hline \multirow{2}{*}{ Gender } & Male: $52 \%$ & Male: $52 \%$ \\
\hline & Female: $48 \%$ & Female: $48 \%$ \\
\hline Average age & 46 years old & 45 years old \\
\hline \multirow{3}{*}{ Labor status } & Unemployed: $36 \%$ & Unemployed: $34 \%$ \\
\hline & Retired: $33 \%$ & Retired: 29\% \\
\hline & Employee: $22 \%$ & Employee: $26 \%$ \\
\hline \multirow{3}{*}{ Marital status } & Single: $50 \%$ & Single: $40 \%$ \\
\hline & Married: $22 \%$ & Married: $25 \%$ \\
\hline & Divorced: $21 \%$ & Divorced: $21 \%$ \\
\hline $\begin{array}{l}\text { History of } \\
\text { substance } \\
\text { abuse }\end{array}$ & $51 \%$ & $51 \%$ \\
\hline
\end{tabular}

\section{RESULTS (cont.)}

The table 2 outlines the socio-demographic profile of the two groups of patients. There were no significant differences between the two regarding gender (in both groups males accounted for $52 \%$ of the sample); average age (46 years old for patients born in Portugal versus 45 years old for Patients born outside of Portugal); labor and marital status (the majority being single and unemployed in both groups); and history of substance abuse (51\% in both groups, particularly alcohol, tabacco and cannabis).

GRAPHIC 3: COMPULSORY AND VOLUNTARY ADMISSIONS ACCORDING TO PLACE OF BIRTH
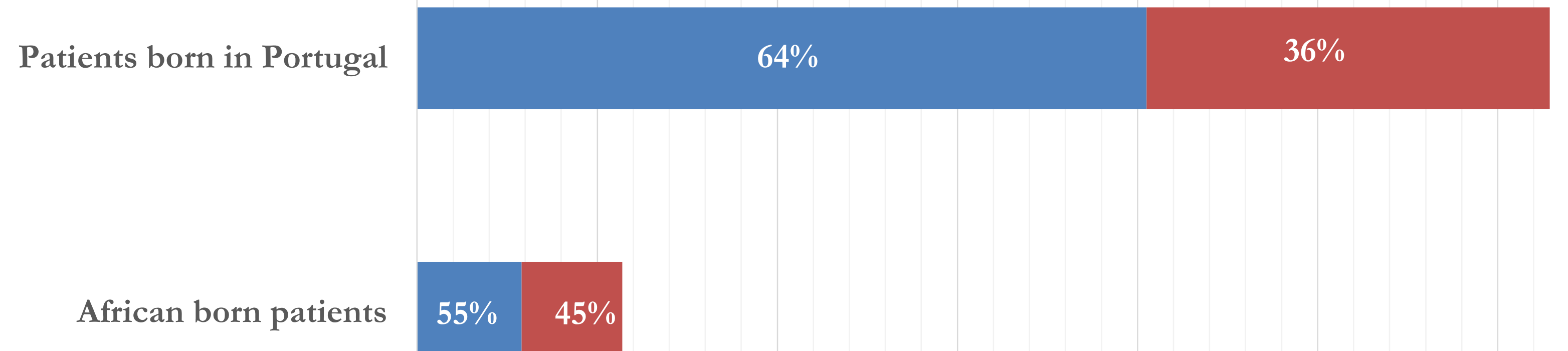

The African born sample presented a slight increase in the length of stay ( 25 versus 22 days) as well as a higher need for compulsory hospitalization ( $45 \%$ versus $36 \%$ ).

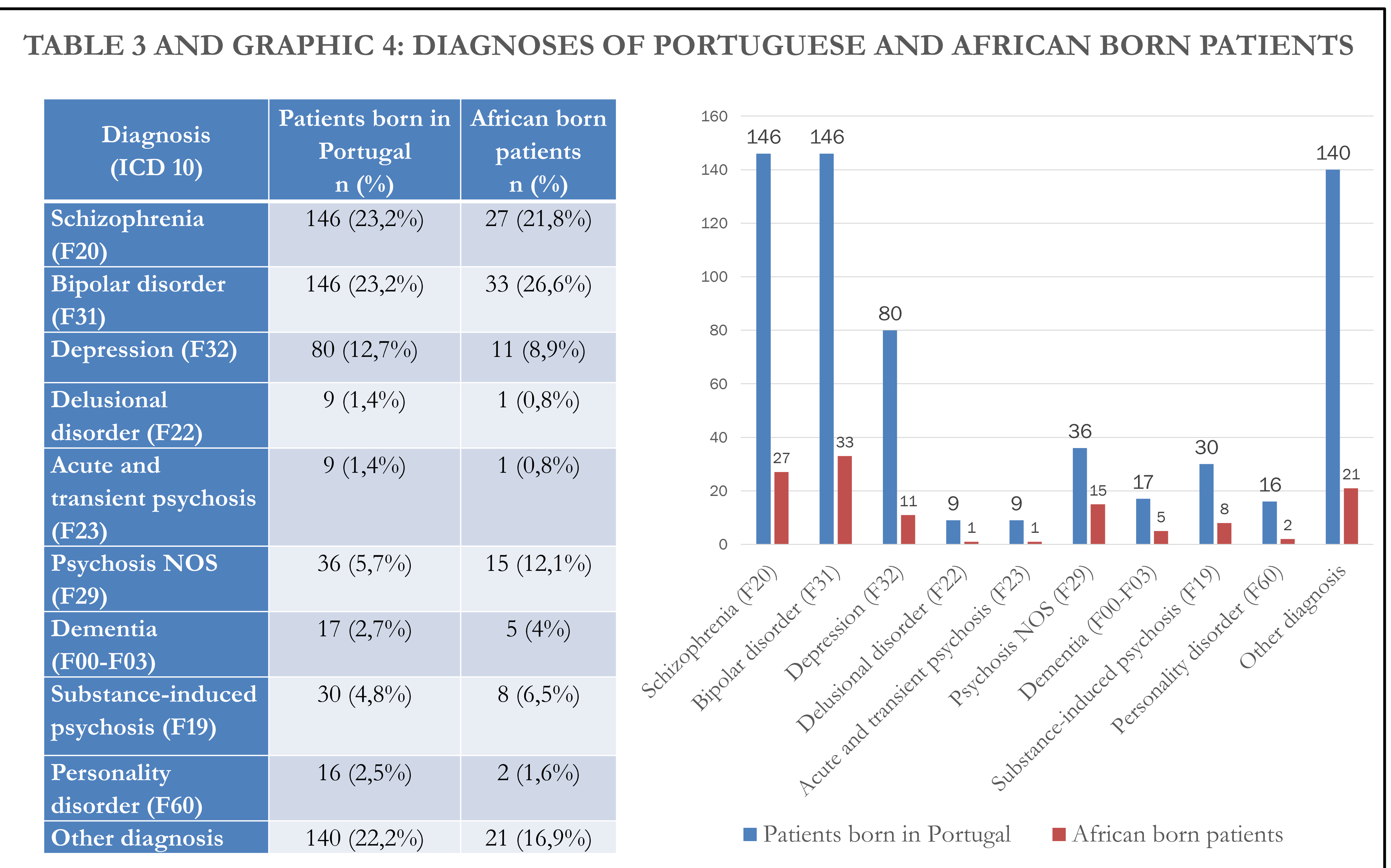

Both groups exhibithed bipolar disorder and schizophrenia as the main diagnosis, with personality disorders being a rare finding. However African born patients did have more psychosis NOS and significantly less depression.

\section{CONCLUSIONS}

Several studies have reported that patients' ethnicity influences psychiatric diagnosis and may correlate with certain clinical characteristics.

The ethnic distribution of the inpatient unit population vastly differs from the population of the hospital catchment area: $84 \%$ of psychiatric inpatients are African, a figure higher than the percentage of African immigrants in Almada-Seixal registered in the 2017 census (40\%). On the other hand, Brazilian immigrant population was under-represented in the inpatient population ( $7 \%$ versus $25,7 \%)$

The sociodemographic profile of the migrant inpatient is a African born, middle-aged single man unemployed, with comorbid substance use, who needs inpatient treatment for about 25 days. - Regarding the clinical profile, and unlike similar studies in current literature, we did not find predominance of schizophrenia in the African born population.

However, we do recognize that our inability to use the ethnic criterion (and our choice of the proxy) may have left out important data, as many patients which are ethnically African were born in Portugal, namely the second-generation, which are known to be at increased risk for severe mental disorders.

At the same time, we should consider that there are some clinical and cultural bias, as the differences in symptom expression can contribute to misdiagnosed psychotic symptoms in a different cultural background (dissociative symptoms, personality traits and cultural beliefs).

- The results of this study highlight the need for a more culturally sensitive care in inpatients units, in order to approach patients according to their demographic and clinical profile, that way improving the quality of health care.

\section{REFERENCES}

1. M. Alda Diez1 J. Garcia Campayo2 N. Sobradiel. (2010). Differences in the diagnosis and treatment of immigrant and local psychiatric inpatients admitted to a general hospisi Th in patients with psychosis: A retrospective medical chart review. Journal of Clinical Psychiatry, 69 (6), 941-945.; 4. Li, D., Law, S., Andermann, L. (2012). Association between degrees of social defeat and themes of delusion in patients with schizophrenia from immigrant and ethnic minority backgrounds. Transcultural Psychiatry 49(5):735-49:;

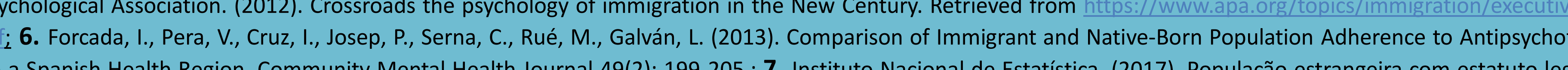

